\title{
Estructura poblacional y distribución espacial del roble negro (Colombobalanus excelsa, Fagaceae), especie endémica, en fragmentos de bosque andino colombiano
}

\author{
Natalia Aguirre-Acosta ${ }^{1} \&$ Jorge Eduardo Botero-Echeverri ${ }^{2}$ \\ 1. Instituto Mulstidisplinario de Biología Vegetal IMBIV-CONICET, C.C. 495, C.P. 5000. Córdoba Argentina; \\ natalia.aguirre@ucaldas.edu.co \\ 2. Centro Nacional de Investigaciones de café CENICAFE, Disciplina Recursos Naturales y Conservación. A.A. 2427 \\ Manizales, Colombia; jorge.botero@cafedecolombia.com
}

\author{
Recibido 08-X-2017. Corregido 09-I-2018. Aceptado 02-II-2018.
}

\begin{abstract}
Population structure and spatial distribution of black oak (Colombobalanus excelsa, Fagaceae), endemic species, in Colombian Andean forest fragments. The Colombian Andean forests are recognized for its great biodiversity and endemism; however, they have undergone a severe process of fragmentation due to anthropic factors. As a result, many vulnerable species have been especially affected. That is the case of the black oak (Colombobalanus excelsa (Lozano, Hern. Cam., \& Henao) Nixon \& Crepet), an endemic and poorly studied species of the Colombian Andes, which has a distribution restricted to only four areas in the country, one of which is the Southeastern Huila region. To examine black oak populations in Serranía Peñas Blancas (Huila), in 2006 we studied the age structure and spatial distribution in plots of 50 x $20 \mathrm{~m}$ (1.6 ha) in forest fragments, in southern distribution. In total, 1228 black oak species were registered among saplings, juvenile and adults, and they have distribution patterns Gamma and Weibull. These results confirm that the majority of the individuals are in the first diametric class. Also, these populations show an added distribution pattern due to the wood extraction process. These results indicate that black oak populations are in a recovery phase after forceful anthropic intervention. Knowing the current status of black oak forests can contribute to the design of effective conservation plans of this species in Colombia. Rev. Biol. Trop. 66(2): 486-494. Epub 2018 June 01.
\end{abstract}

Key words: endemic species; vulnerable tree; conservation; anthropic disturbance; restricted distribution.

Los bosques andinos son sitios reconocidos como hotspots de biodiversidad por su alto grado de endemismo y alta riqueza de especies (Orme et al., 2005). Sin embargo, estos han sido fuertemente sometidos a presiones antrópicas como la deforestación, la ampliación de la frontera agrícola, el desarrollo urbanístico y la extracción o saqueo de madera, lo que ha conllevado a una pérdida del $73 \%$ de su cobertura original desde 1980 hasta el 2006 (Cavelier \& Etter, 1993; Etter, Mcalpine, Wilson, Phinn, \& Possingham, 2006). Generalmente estos cambios en la cobertura arbórea, van acompañados de un proceso de fragmentación, cuya principal consecuencia es la formación de parches de bosque de diferentes tamaños aislados entre si, dando como resultado una alteración de la estructura, distribución y funcionamiento de las comunidades vegetales, además de una reducción de los tamaños poblaciones de las plantas nativas (Saunders, Hobbs, \& Margules, 1991; Fahrig, 2003; Etter et al., 2006).

Esta fragmentación del bosque, puede afectar de manera particular poblaciones de especies endémicas con distribuciones restringidas y sistemas reproductivos complejos. Este es el caso del roble negro (Colombobalanus excelsa (Lozano, Hern. Cam. \& Henao) Nixon \& Crepet)), especie endémica de los bosques andinos colombianos que presenta un sistema 
sexual monoico, con inflorescencias unisexuales, baja tasa de germinación y bajo reclutamiento de nuevos individuos (Cárdenas \& Salinas, 2006; Palacio-Mejía \& Fernández-M, 2006; Parra-Aldana, Diez-Gómez, \& MorenoHurtado, 2011). Su distribución ha quedado restringida a solo cuatro localidades, situadas entre 1400 y 2200 m.s.n.m en las tres cordilleras andinas. Particularmente en estas altitudes, los cambios en la cobertura original del bosque se han dado principalmente para el establecimiento de café y otros cultivos como actividad económica, y en los últimos años la pérdida de bosque en estos sitios, se ha relacionado con la ganadería extensiva y el crecimiento urbano (Etter et al., 2006; Forero, 2010).

Por lo tanto, el objetivo de este estudio se centró en evaluar la estructura poblacional y la distribución espacial de poblaciones de roble negro en cuatro fragmentos de bosque situados en la Serranía de Peñas Blancas-Huila, distribución más sur de la especie, con la finalidad de aportar conocimiento de su dinámica ecológica. Actualmente el roble negro está catalogado como vulnerable a la extinción en estado silvestre, debido a la fragmentación de bosque y al saqueo selectivo de su madera (Cárdenas \& Salinas, 2006; Ministerio de Ambiente, Vivienda y Desarrollo Territorial, 2010). Según Devia y Arenas (1995) y Calderón (2001), la vulnerabilidad de esta especie está asociada particularmente a que es un árbol emergente del bosque, con características que lo hacen propicio para ser utilizado como materia prima por los aserradores locales, ya que puede alcanzar una altura entre 20 y $40 \mathrm{~m}$, con copa globosa y fuste recto, de más de un metro de diámetro a la altura del pecho y una madera de alta dureza (Parra-Aldana et al., 2011). Es por esto que se espera encontrar poblaciones aisladas, en fase de recuperación, además de distribuciones no aleatorias de los individuos en cada fragmento de bosque, ya que en la zona de estudio se presenta tala selectiva y un fuerte proceso de fragmentación de bosque.

\section{MATERIALES Y MÉTODOS}

Área de estudio: Los cuatro fragmentos de bosque estudiados La Palma, La Marimba, Alto Santa Bárbara y Alto Bellavista, se ubican en la parte más alta de la Serranía de Peñas Blancas (i.e., alturas comprendidas entre 1630 y $1900 \mathrm{~m}$ ), en el extremo suroriental del departamento del Huila (Apéndice digital 1), área más sur de distribución de la especie. Esta zona corresponde al bosque sub-andino (Cleef, Rangel, Van der Hammen, \& Jaramillo, 1984), comprendendiendo zonas de montaña con pendientes marcadas. La temperatura promedio de la región varía entre 16 y $20^{\circ} \mathrm{C}$, la precipitación presenta un pico máximo de lluvias en el mes de julio de $235 \mathrm{~mm}$ y uno mínimo en el mes de enero de $63 \mathrm{~mm}$ (Eslava, López, \& Olaya, 1986).

\section{Muestreo}

Estructura poblacional: En cada fragmento de bosque se delimitaron cuatro parcelas de $50 \times 20 \mathrm{~m}\left(1000 \mathrm{~m}^{2}\right.$, Urrego \& González 2000; Vallejo, Londoño-V., López-C., Galeano, Álvarez-D., \& Devia, 2005) durante el año 2006, con un área total medida de $4000 \mathrm{~m}^{2}(0.4$ ha). En cada una, se midieron todos los individuos de roble negro sin discriminar su diámetro a la altura del pecho (DAP). Se clasificaron como: plántulas a los individuos con una altura $\leq 1.3 \mathrm{~m}$, a los cuáles no se les midió DAP, sino únicamente su altura. Juveniles a los individuos con un DAP $\leq 9.9 \mathrm{~cm}$, adultos a los individuos con DAP $\geq 10 \mathrm{~cm}$ (Caldato, Vera, \& Mac, 2002). Dentro de cada fragmento de bosque, las parcelas fueron ubicadas aleatoriamente con la finalidad de obtener la mayor variabilidad local posible. Posteriormente, las cuatro parcelas por fragmento de bosque fueron sumadas a una unidad de análisis, con la finalidad de obtener la estructura etaria por fragmento de bosque y no por parcela, ya que el interés particular era comparar los diferentes fragmentos de bosque; quedando así un área total evaluada por fragmento de bosque de $4000 \mathrm{~m}^{2}$ (0.4 ha). 
Distribución espacial: Para determinar la distribución espacial del roble negro en cada parcela de $1000 \mathrm{~m}^{2}$, cada individuo fue ubicado en un plano cartesiano, donde $\mathrm{X}$ indicaba una distancia de 0 a $20 \mathrm{~m}$ y Y de 0 a $50 \mathrm{~m}$ (Alder \& Synnot, 1992; Vallejo et al., 2005). Para la distribución espacial tambien fueron sumadas las cuatro parcelas por fragmento de bosque, donde $\mathrm{X}$ indica una distancia de 0 a $40 \mathrm{~m}$ y $\mathrm{Y}$ una distancia de 0 a $100 \mathrm{~m}$.

\section{Análisis de datos}

Estructura etaria: Por medio del programa Infostat versión 2012 (INFOSTAT, 2004), se obtuvieron modelos probabilísticos de distribución de frecuencias diamétricas de todos los individuos en cada uno de los cuatro remanentes de bosque. Para incluir en los análisis los individuos a los que no se les midió DAP (i.e., plántulas), se transformaron los datos sumando uno a cada diámetro (DAP +1$)$, luego se dividió el total de individuos en ocho clases diámetricas. Para la obtención de los modelos, se utilizaron frecuencias relativas. La frecuencia relativa se entiende como el cociente entre la frecuencia absoluta y el tamaño de la muestra. Para determinar el ajuste del mejor modelo teórico de distribución de frecuencias diamétricas, se realizó la prueba de bondad de ajuste Kolmorogov-Smirnov (Johnson, Kotz, \& Balakrisnan, 1995). Por último, se comparó la densidad de individuos por hectárea y el DAP promedio de cada fragmento por medio de la prueba $\chi^{2}$ (Zar, 1999). La proporción de individuos por clase diamétrica se comparó por medio de una prueba Wilcoxon no paramétrica (Zar, 1999).
Distribución espacial: Se aplicó la metodología de análisis espacial por índices de distancia, SADIE (Spatial analysis by distance Indices) por sus siglas en inglés (Perry, 1995). Con esta metodología cada individuo recibió una posición espacial a través de sus coordenadas cartesianas $(\mathrm{X}, \mathrm{Y})$ y estos se analizaron a través del indice de agregracion Ia (Perry, 1995). Donde, valores de $I a>1$ indican distribución agregada, $I a<1$ indican distribución uniforme, y $I a=1$ indican distribución aleatoria (Perry, Bell, Smith, \& Woiwoid, 1996; Perry, 1998). Estos análisis fueron desarrollados con el programa SADIEShell versión 2 (Perry et al., 1996; Conrad, Perry, Woiwoid, \& Alexander, 2006).

\section{RESULTADOS}

Estructura poblacional: En total fueron censados 1228 individuos en los cuatro fragmentos de bosque en 1.6 ha ( 0.4 has por fragmento de bosque). Encontrándose 105 individuos en Alto Bellavista, 399 en la Palma, 505 en Alto Santa Bárbara y 218 en La Marimba. Al llevar esta densidad de individuos por hectárea, la densidad varío entre 262.5 ha en Alto Bellavista, 997.5 ha en la Palma, 1265 ha en Alto Santa Bárbara y 545 ha en La Marimba, encontrándose diferencias significativas entre los fragmentos de bosque $\left(\chi^{2}=788.19\right.$, g.l. $=$ $3 \mathrm{p}<0.001)$. Asimismo el DAP promedio por fragmento varió entre 15.22 y 39.27 , lo que también evidencia diferencias significativas entre fragmentos de bosque $\left(\chi^{2}=15.35\right.$, g.l. $=3$ $\mathrm{p}<0,005$; Cuadro 1).

CUADRO 1

Individuos totales medidos en cada fragmento de bosque

TABLE 1

Total individuals measured in each forest fragment

\begin{tabular}{lccc}
\multicolumn{1}{c}{ Fragmento de Bosque } & Ind. totales & Ind. Ha & DAP prom. \\
Alto Bellavista & 105 & 262.5 & 39.27 \\
La Palma & 399 & 997.5 & 15.22 \\
Alto Santa Bárbara & 506 & 1265 & 17.14 \\
La Marimba & 218 & 545 & 22.10 \\
\hline
\end{tabular}

Ha: hectárea, DAP: dimátro a la altura del pecho promedio para cada fragmentos de bosque. 
CUADRO 2

Densidades de los individuos de roble negro para los cuatro fragmentos de bosque

TABLE 2

Densities of black oak individuals for the four forest fragments

\begin{tabular}{|c|c|c|c|c|c|c|c|}
\hline \multicolumn{4}{|c|}{ Alto Bellavista } & \multicolumn{4}{|c|}{ Alto Santa Bárbara } \\
\hline $\begin{array}{c}\text { Clases } \\
\text { diamétricas }\end{array}$ & $\begin{array}{c}\text { Frecuencia } \\
\text { absoluta }\end{array}$ & $\begin{array}{c}\text { Frecuencia } \\
\text { relativa }\end{array}$ & $\begin{array}{c}\text { Individuos } \\
\text { por } \mathrm{Ha}\end{array}$ & $\begin{array}{c}\text { Clases } \\
\text { diamétricas }\end{array}$ & $\begin{array}{c}\text { Frecuencia } \\
\text { absoluta }\end{array}$ & $\begin{array}{c}\text { Frecuencia } \\
\text { relativa }\end{array}$ & $\begin{array}{c}\text { Individuos } \\
\text { por Ha }\end{array}$ \\
\hline $1-35.88$ & 62 & 0.59 & 155.00 & $1-25.75$ & 406 & 0.8 & 1015 \\
\hline $35.88-70.75$ & 20 & 0.19 & 50.00 & $25.75-50.5$ & 62 & 0.12 & 155 \\
\hline $70.75-105.63$ & 13 & 0.12 & 32.50 & $50.5-75.25$ & 28 & 0.06 & 70 \\
\hline $105.63-140.5$ & 8 & 0.08 & 20.00 & $75.25-99.99$ & 5 & 0.01 & 12.5 \\
\hline $140.5-175.38$ & 2 & 0.02 & 5.00 & $99.99-124.74$ & 4 & 0.01 & 10 \\
\hline $175.38-210.25$ & 0 & 0 & 0 & $124.74-149.49$ & 0 & 0 & 0 \\
\hline $210.25-245.13$ & 0 & 0 & 0 & $149.49-174.24$ & 0 & 0 & 0 \\
\hline $245.13-280$ & 0 & 0 & 0 & 174.24-198.99 & 1 & 0 & 2.5 \\
\hline total & 105 & 1 & 262.5 & total & 506 & 1 & 1265 \\
\hline \multicolumn{4}{|c|}{ La Palma } & \multicolumn{4}{|c|}{ La Marimba } \\
\hline $\begin{array}{c}\text { Clases } \\
\text { diamétricas }\end{array}$ & $\begin{array}{c}\text { Frecuencia } \\
\text { absoluta }\end{array}$ & $\begin{array}{c}\text { Frecuencia } \\
\text { relativa }\end{array}$ & $\begin{array}{c}\text { Individuos } \\
\text { por Ha }\end{array}$ & $\begin{array}{c}\text { Clases } \\
\text { diamétricas }\end{array}$ & $\begin{array}{c}\text { Frecuencia } \\
\text { absoluta }\end{array}$ & $\begin{array}{l}\text { Frecuencia } \\
\text { relativa }\end{array}$ & $\begin{array}{c}\text { Individuos } \\
\text { por } \mathrm{Ha}\end{array}$ \\
\hline $1-34.82$ & 348 & 0.87 & 870 & $1-23.2$ & 153 & 0.7 & 382.5 \\
\hline $34.82-68.64$ & 35 & 0.09 & 87.5 & $23.2-45.4$ & 28 & 0.13 & 70 \\
\hline 68.64-102.46 & 9 & 0.02 & 22.5 & $45.4-67.61$ & 10 & 0.05 & 25 \\
\hline $102.46-136.28$ & 4 & 0.01 & 10 & $67.61-89.81$ & 13 & 0.06 & 32.5 \\
\hline $136.28-170.1$ & 0 & 0 & 0 & $89.81-112.01$ & 5 & 0.02 & 12.5 \\
\hline $170.1-203.92$ & 1 & $2.50 \mathrm{E}-03$ & 2.5 & $112.01-134.21$ & 4 & 0.02 & 10 \\
\hline $203.92-237.74$ & 0 & 0 & 0 & $134.21-156.41$ & 4 & 0.02 & 10 \\
\hline $237.74-271.56$ & 2 & 0.01 & 5 & $156.41-178.62$ & 1 & $4.60 \mathrm{E}-03$ & 2.5 \\
\hline Total & 399 & 1 & 997.5 & Total & 218 & 1 & 545 \\
\hline
\end{tabular}

La mayor proporción de individuos en los cuatro fragmentos de bosque, se encuentra en las tres primeras clases diamétricas. Alto Bellavista presentó en su primer clase diamétrica $59 \%$ del total de los individuos censados, Alto Santa Bárbara $80 \%$, La Palma 87 \% y La Marimba $70 \%$ respectivamente (Cuadro 2). La comparación entre fragmentos de bosque, evidencia que no existen diferencias significativas entre la proporción de individuos en cada clase diamétrica $(\mathrm{W}=6.02, \mathrm{p}=0.1)$, independientemente de la cantidad de individuos encontrados en cada fragmento de bosque.

Modelos probabilísticos y estructura etaria: Para cada uno de los fragmentos de bosque se obtuvo un modelo de distribución de frecuencias diamétricas (Apéndice digital
2, Fig. 1). Estos modelos tuvieron dos tipos de ajustes para los cuatro fragmentos de bosque estudiados, así el modelo probabilístico de distribución de frecuencias diamétricas con mayor ajuste fue el Gamma, para los fragmentos de bosque Bellavista, La Palma y La Marimba; y, el modelo Weibull, para el fragmento de bosque Alto Santa Bárbara (Fig. 1). Los modelos Gamma y Weibull que se ajustaron a cada fragmento, sugieren que los bosques pertenecen a rodales multietáneos.

Distribución espacial del roble negro en cada remanente de bosque: Todos los fragmentos presentaron distribuciones espaciales agregadas $I a>1$ aunque con diferentes valores de significancia, así: Alto Bellavista ( $\mathrm{Ia}=1.48$, $\mathrm{p}=0.04)$ y La Marimba $(\mathrm{Ia}=1.87, \mathrm{p}=0.003)$ 
La Palma

Ajuste: Gama $(0.038,0.579)$

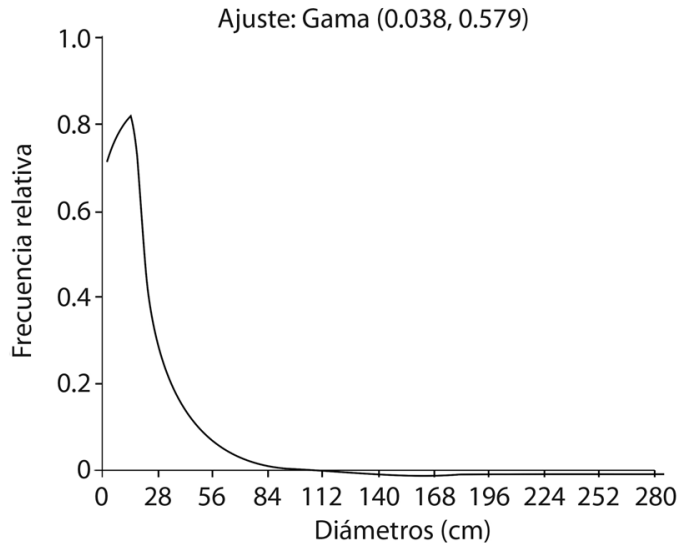

Alto Santa Bárbara

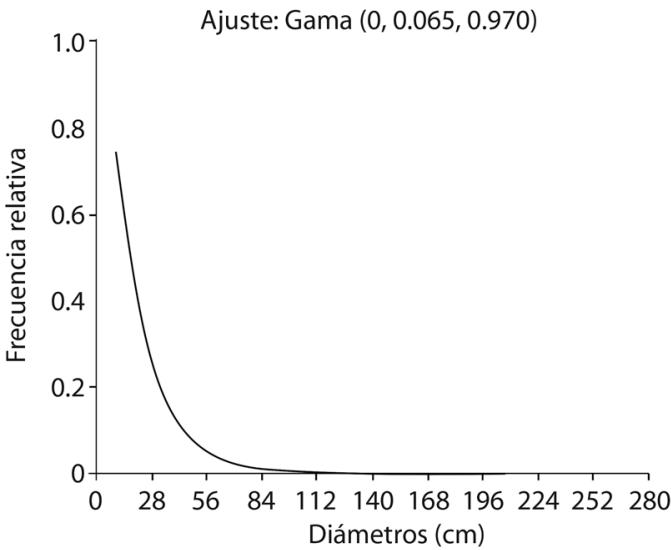

Bellavista

Ajuste: Gama $(0.017,0.676)$

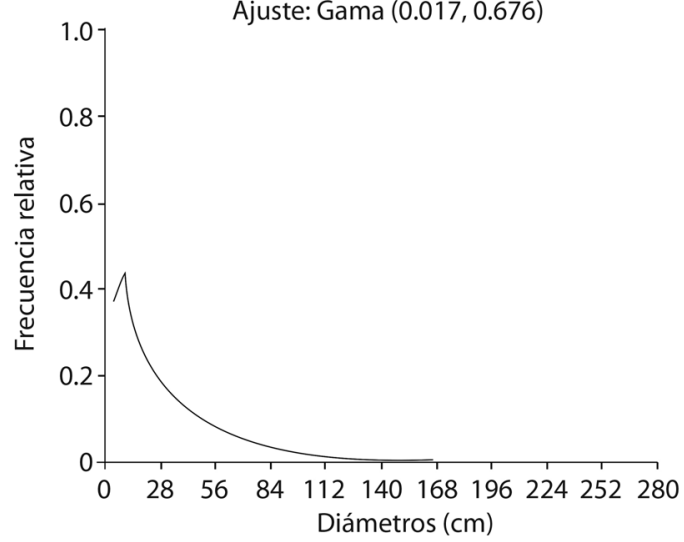

La Marimba

Ajuste: Gama $(0.038,0.579)$

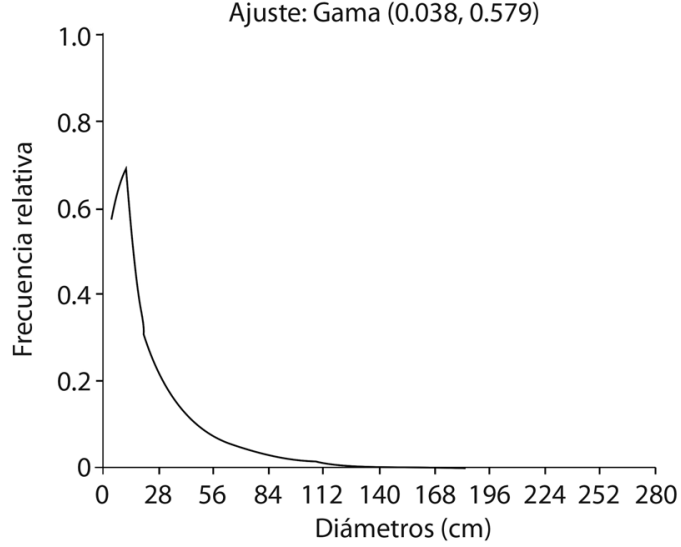

Fig. 1. Modelos probabilísticos de distribución de frecuencias diamétricas en individuos de roble negro, para los cuatro fragmentos de bosque intervenido.

Fig. 1. Probabilistic models of diametrical frequency distribution in black oak individuals, for the four forest fragments.

presentaron una distribución agregada estadísticamente significativa y, La Palma $(\mathrm{Ia}=10.97$, $\mathrm{p}=0.0002)$ y Alto Santa Bárbara $(\mathrm{Ia}=10.85$, $\mathrm{p}=0.0002)$ presentaron una distribución agregada altamente significativa.

\section{DISCUSIÓN}

Existe una gran variación en la densidad de individuos por hectárea para cada uno de los fragmentos de bosque. Esta gran variación podría ser el resultado de diferentes procesos de regeneración y establecimiento después de diferentes intensidades de tala o saqueo de madera. Por ejemplo, en el fragmento de bosque de Alto bellavista, el cual es protegido por la comunidad local y tuvo una menor intensidad de saqueo, la densidad fue de $262.5 \mathrm{ind} /$ ha, en contraste con el fragmento de bosque de Alto Santa Bárbara donde la densidad fue de $1265 \mathrm{ind} /$ ha siendo el fragmento de bosque más intervenido. Estos resultados coinciden con lo encontrado en otros estudios realizados sobre la misma especie y en bosques cercanos a los sitios de estudio. Por ejemplo Parra-Aldana et al. (2011), en poblaciones muy intervenidas 
encontraron una densidad de 430 ind/ha y Dávila, Alvis y Ospina (2012) encontraron una densidad de $281.7 \mathrm{ind} / \mathrm{ha}$ en poblaciones menos intervenidas que se encuentran en el Parque Nacional Natural Cueva de los Guácharos.

En general el mayor porcentaje de individuos en todos los fragmentos de bosque se encuentra en las primeras clases diamétricas (Parra-Aldana et al., 2011; Dávila et al., 2012; Moreno, 2014) y este porcentaje es proporcional a la densidad de individuos por hectárea, e.g., Alto Bellavista tiene el $59 \%$ del total de los individuos en la primer clase diametrica y Alto Santa Bárbara tiene el $80 \%$. Estos resultados indican, que estos bosques en general se encuentran en una fase de recuperación después de una fuerte intervención antrópica de diferentes intensidades en cada fragmento de bosque.

Encontrar un alto porcentaje de individuos en la primera clase diamétrica, indica que se puede estar facilitando el establecimiento de plántulas en los cuatro fragmentos de bosque, y que éste establecimiento es mayor en bosques más saqueados o talados. Probablemente existe mayor establecimiento debido a que el roble negro es una especie heliófita en sus primeros estadios de desarrollo, es decir, plántulas y árboles jóvenes necesitan de luz directa para su crecimiento y desarrollo (Nixon \& Crepet, 1989; Parra-Aldana et al., 2011). Por otra parte, encontrar una alta densidad de individuos en la primera clase diamétrica en los cuatro fragmentos de bosque, permite inferir que existe una reserva de individuos jóvenes que más adelante podrían reemplazar a los árboles de mayor tamaño que vayan desapareciendo (Villa, 2005), ya sea por muerte natural o por extracción selectiva. Asimismo, la alta reserva de individuos en las primeras clases diamétricas, indica que de cierta forma la explotación selectiva de bosques de roble ha sido una herramienta eficaz para promover su regeneración natural (Guariguata \& Saenz, 2002; Guerrero, Paz, \& Parrado, 2010).

Sin embargo, estas conclusiones deben tomarse con precaución, ya que en este trabajo no se evaluó como varía el porcentaje de establecimiento y viabilidad de semillas. Por ejemplo, Parra-Aldana et al. (2011) encontraron un bajo establecimiento y una baja viabilidad de las semillas de la especie y Moreno (2014), encontró que la baja viabilidad de las semillas estaba asociada a una alta depredación de estas en el banco de semillas. Otros trabajos también han mencionado la dificultad de regeneración de diferentes especies de robles (González, Ochoa, Ramírez, \& Quintana, 1995; Pulido, 2002), estableciendo que la falta de reclutamiento puede conducir a la disminución a largo plazo de las poblaciones naturales (Sork et al., 2002).

Los modelos de distribución de frecuencias diamétricas, los cuales incluyen la cantidad de individuos por clases de tamaños o clases diamétricas dentro de una población, son buenos predictores del estado actual de poblaciones con algún grado de amenaza, como lo es el roble negro. En este trabajo, en tres fragmentos de bosque se ajustaron modelos de distribución de frecuencias diamétricas Gamma y para el fragmento de bosque Alto Santa Bárbara, que presentó mayor disturbio antrópico, se ajustó un modelo Weibull. Esta última población (i.e., Alto Santa Bárbara), presentó mayor cantidad de individuos totales $(\mathrm{N}=506)$ y mayor porcentaje de individuos en las primeras clases diamétricas $(80 \%$ de individuos en la primera clase diamétrica), en relación a los otros tres bosques fragmentados, lo cuál hizo que la curva del modelo tuviera una pendiente mucho más pronunciada. En general, estos modelos representan poblaciones con distribuciones en $\mathrm{J}$ invertida, que indican que se trata de poblaciones disetáneas con estructuras de tamaños aparentemente balanceadas, en las cuales existe una mayor cantidad de individuos en las categorías diamétricas menores.

La distribución espacial fue agregada (Ia $>$ 1) para cada uno de los cuatro fragmentos de bosque, siendo altamente significativa para La palma y Alto Santa Bárbara. La distribución espacial podría estar relacionada a la densidad de individuos encontrados en las primeras clases diamétricas y esta alta densidad en las primeras clases, a su vez puede estar relacionado a un alto establecimiento de plántulas en 
zonas de clareo después de un proceso de tala selectiva de individuos adultos de gran porte. La tala selectiva se practicó intensivamente en la zona de estudio en los años 80 .

Se puede concluir que encontrar una estructura etaria que corresponde con poblaciones en $\mathrm{J}$ invertida y con distribuciones espaciales agregadas, que a su vez se ajustan a modelos de distribución diamétrica Gamma y Weibull; son poblaciones que se encuentran en fase de recuperación después de una fuerte intervención antrópica. Esta intervención ha afectado de manera más drástica a las poblaciones de los fragmentos La Palma y Alto Santa Bárbara y en menor medida a las poblaciones de los fragmentos Alto Bellavista y La Marimba. Sin embargo, esta intervención antrópica ha permitido que exista un establecimiento de plántulas en zonas de clareo, que de no existir probablemente la estructura etaria correspondería a poblaciones con mayor cantidad de individuos adultos, poblaciones clímax donde el establecimiento tiende a ser minimo por la estructura de edades de los individuos (Crawley, 2003).

Finalmente, una de las consecuencias más agresivas de la fragmentación es la disminución del tamaño poblacional y los procesos que estos conllevan como son la endogamia y la disminución del tamaño poblacional efectivo. Esta especie aunque presenta una gran cantidad de plántulas y juveniles que aseguran su permanencia en el tiempo, también presenta baja diversidad genética (Aguirre-Acosta, Palacio-Mejía, Barrios-Leal, \& Botero-Echeverri, 2013; Palacio-Mejía \& Fernández-M., 2006), lo que podría ocasionar una progresiva disminución de la capacidad reproductiva de la especie (Swaine, Lieberman, \& Putz, 1987; Parra-Aldana et al., 2011) y a su vez, una extinción a largo plazo. Es por esto, que el conocimiento que se pueda generar en cuanto a la dinámica poblacional del roble negro en cada una de las localidades donde aún existe, sirve como insumo para generar planes de manejo y conservación en conjunto con las comunidades locales.

\section{AGRADECIMIENTOS}

Agradecemos las observaciones realizados por dos revisores anónimos, a Jorge Paiba y Juan David Corrales por su ayuda en la toma de datos en campo y a las comunidades cafeteras por todo el apoyo logístico en los sitios de muestreo. Este trabajo estuvo enmarcado dentro del proyecto: Valoración de los servicios ambientales de la biodiversidad en zonas cafeteras (BDC 0304) programa Biología de la Conservación del Centro Nacional de Investigación en Café, CENICAFE. NAA desarrolló este trabajo como estudiante de pregrado de la Universidad de Caldas; JEBE es investigador de la disciplina de Recursos Naturales y Conservación de CENICAFE.

\section{RESUMEN}

Los bosques andinos en Colombia son reconocidos por su alta biodiversidad y endemismo, sin embargo han experimentado un severo proceso de fragmentación debido a procesos antrópicos. Como resultado de esto, muchas especies vulnerables han sido particularmente afectadas. Este es el caso del roble negro (Colombobalanus excelsa (Lozano, Hern. Cam., \& Henao) Nixon \& Crepet), especie endémica y poco estudiada de los Andes colombianos, cuya distribución está restringida a cuatro localidades en el país, una de ellas el sector suroriental del departamento del Huila. Con el fin de estudiar las poblaciones de roble negro en la Serrania de Peñas Blancas (Huila), localidad más sur de distribución; en 2006 se establecieron 16 parcelas de 50 x $20 \mathrm{~m}$ (1.6 ha) en fragmentos de bosque, donde se determinó la estructura etaria y la distribución espacial de esta especie. En total se registraron 1228 individuos de roble negro entre adultos, juveniles y plántulas; los cuáles presentaron patrones de distribución Gamma y Weibull. Con estos resultados se pudo confirmar que la mayoría de los individuos se encuentran en las primeras clases diamétricas. Asimismo, sus poblaciones presentaron un patrón de distribución agregado debido al proceso de extracción de madera. Estos resultados indican que las poblaciones de roble negro se encuentran en una fase de recuperación despúes de una fuerte intervención antrópica. El conocimiento del actual estado de los bosques de roble negro, puede contribuir para el diseño de efectivos planes de conservacion de la especie en Colombia.

Palabras clave: especie endémica; árbol vulnerable; conservación; disturbio antrópico; distribución restringida. 


\section{REFERENCIAS}

Aguirre-Acosta, N., Palacio-Mejía, J. D., Barrios-Leal, D. J., \& Botero Echeverri, J. E. (2013). Diversity and Genetic Structure of the Monotypic Genus Colombobalanus (Fagaceae) in Southeast of Colombian Andeans. Caldasia, 35(1), 123-133.

Alder, D., \& Synnott, T. J. (1992). Permanent sample plot techniques for mixed tropical forests. Tropical Forestry Papers (pp. 124). Oxford Forestry Institute.

Caldato, S. L., Vera, N., \& Mac, D. P. (2002). Estructura poblacional de Ocotea puberula en un bosque secundario y primario de la selva mixta misionera. Ciencia forestal, Santa María, 13(1), 25-32.

Calderón, E. (2001). Plantas colombianas en peligro, extintas o en duda. Instituto Alexander von Humboldt. Bogotá: Colombia.

Cárdenas, L. D. \& Salinas, N. (2006). Libro rojo de plantas de Colombia. Especies maderables amenazadas, parte 1. Sinchi, (pp. 169). Instituto Amazónico de investigaciones biológicas y Ministerio de medio ambiente, vivienda y desarrollo territorial.

Cavelier, J., \& Etter, A. (1993). Deforestation of Montane Forest in Colombia as a Result of Illegal Plantations of Opium. In. Biodiversity and Conservation of Neotropical Montane Forest (pp. 541-549). Edited by Churchill, S., Balslev, H., Forero, E., \& Luteyn J. (1993). The New York Botanical Garden.

Cleef, A. M., Rangel, O. C., Van Der Hammen, T., \& Jaramillo M. (1984). La vegetación de las selvas del transecto Buritaca. En: T. van der Hammen \& P.M. Ruiz, Eds. La Sierra Nevada de Santa Marta (Colombia), transecto Buritaca-La Cumbre. Studies on Tropical Andean Ecosystems, (pp. 267-406). Berlin-Stuttgart: J. Cramer.

Conrad, K. F., Perry, J. N., Woiwoid, I. P., \& Alexander, C. J. (2006). Large-scale temporal changes in spatial pattern during declines of abundance and occupancy in a common moth. Journal of Insect Conservation, $10,53-64$.

Crawley, M. (2003). Plant Ecology (pp. 717). Second Edition.

Dávila, E. D., Alvis, J. F., \& Ospina, R. (2012). Distribución espacial, estructura y volúmen de los bosques de roble negro (Colombobalanus excelsa (Lozano, Hern. Cam. \& Henao) Nixon \& Crepet) en el Parque Nacional Natural Cueva de los Guácharos. Colombia Forestal, 15(2), 207-214.

Devia-C, C. A., \& Arenas, H. S. (1995). Evaluación del estatus ecosistémico y de manejo de los bosques de fagáceas (Quercus humboldtii y Trigonobalanus excelsus) en el norte de la Cordillera Oriental (Cundinamarca, Santander y Boyacá). Capitulo 3. En. Desarrollo sostenible en los Andes de Colombia (provincias del norte, Gutiérrez y Valderrama) Boyacá Colombia. IDEADE. Pontificia Universidad Javeriana, Unión Europea.

Eslava, J.A., López, V.A., \& Olaya, G. (1986). Contribución al conocimiento del régimen térmico y pluviométrico de Colombia. Colombia Geográfica, 12(2), 53-118.

Etter, A., Mcalpine, C., Wilson, K., Phinn, S., \& Possingham, H. (2006). Regional patterns of agricultural land use and deforestation in Colombia. Agriculture. Ecosystems and Environment, 114, 369-386.

Fahrig, L. (2003). Effects of Habitat Fragmentation on Biodiversity. Annual Review Ecology Evolution and Systematics, 34, 487-515.

Forero, J. (2010). "Colombian Family Farmers" Adaptations to New Conditions in the World Coffee Market. Latin American Perspectives, 37(2), 93-110.

González, M., Ochoa, S., Ramírez, N., \& Quintana, P. (1995). Current land use trends and conservation of old growth forest habitats in the highland of Chiapas, Mexico (pp. 190-198.) In: Wilson M.H and Sader S.A. (Eds). Conservation of neotropical migratory birds in Mexico. Maine Agriculture and Forest Experiment Station, Miscellaneous Publication 727 Orono, Maine.

Guariguata, M., \& Saenz, G. (2002). Post-logging acorn production and oak regeneration in a tropical montane forest, Costa Rica. Forest Ecology and Management, 167(1-3), 285-293.

Guerrero, S. B., Paz, E. S., \& Parrado, A. (2010). Efectos de la intervención antrópica en la distribución de las semillas y plántulas de roble (Quercus humboldtii Bonpl., Fagaceae), en la cordillera oriental colombiana. Colombia Forestal, 13(1), 163-180.

INFOSTAT. (2004). Manual del Usuario. Grupo InfoStat, FCA, Universidad Nacional de Córdoba. (Primera Edición, versión 2004). Editorial Brujas Argentina.

Johnson, N. L., Kotz, S., \& Balakrisnan, N. (1995). Continuous Univariate Distributions. Volume 1 (pp. 756). Second Edition. John Wiley \& Sons, Inc.

Ministerio de Ambiente, Vivienda y Desarrollo Territorial. (2010). Resolución 383 de febrero de 2010. Por la cual se declaran las especies silvestres que se encuentran amenazadas en el territorio nacional y se toman otras determinaciones. Diario oficial No. 47635 de 26 de febrero de 2010.

Moreno, J. S. (2014). Estructura poblacional del roble negro (Colombobalanus excels) en el Valle del Cauca, una herramienta para la formulación de una estrategia para su conservación. (Tesis de pregrado). Universidad Icesi. 
Nixon, K.C., \& Crepet, W. (1989). Trigonobalanus (Fagaceae): Taxonomic Status and Phylogenetic Relationships. American Journal of Botany, 76(6), 828-841.

Orme, C. D. L., Davies, R.G., Burgues, M., Eingenbrod, F., Pickup, N., Olson, V. A., ... \& Owens, I. P. F. (2005). Global hotspots of species richness are not congruent with endemism or threat. Nature, 436, 1016-1019.

Palacio-Mejía, J. D., \& Fernández-M, J. F. (2006). Estado de la investigación en genética de la conservación de los robles (Fagacea) en Colombia. En: Memorias del l Simposio Internacional de Robles y Ecosistemas Asociados (pp. 291) Solano, C y N. Vargas (editoras). Bogotá: Fundación Natura-Pontificia Universidad Javeriana.

Parra-Aldana, C. A., Diez-Gómez, M. C., \& Moreno-Hurtado, F. H. (2011). Regeneración Natural del Roble negro (Colombobalanus excelsa, Fagaceae) en dos Poblaciones de la Cordillera de los Andes, Colombia. Revista Facultad Nacional de Agronomía Medellín, 64(2), 6175-6180.

Perry, J. N. (1995). Spatial analysis by distance indices. Journal of Animal Ecology, 64, 303-314.

Perry, J. N. (1998). Measures of spatial pattern for counts. Ecology, 79, 1008-1017.

Perry, J. N., Bell, E. D., Smith, R. H., \& Woiwoid, I. P. (1996). SADIE: software to measure and model spatial pattern. Aspects of Applied Biology, 46, 95-102.

Pulido, J. F. (2002). Biología reproductiva y conservación: el caso de la regeneración de bosques templados y subtropicales de robles (Quercus spp.). Revista Chilena de Historia Natural, 75, 5-15.
Saunders, D. A., Hobbs, R. J., \& Margules, C. R. (1991). Biological Consequences of Ecosystem. A Review. Conservation Biology, 5(1), 18-32.

Sork, V. L., Davis, F. W., Smouse, P. E., Apsit, V. J., Dyer, R. J., Fernandez, J. F., \& Kuhn, B. (2002). Pollen movement in declining populations of California Valley oak, Quercus lobata: where have all the fathers gone? Molecular Ecology, 11(9), 1657-1668.

Swaine, M. D., Lieberman, D., \& Putz, F. E. (1987). The dynamics of tree populations in tropical forest: a review. Journal of Tropical Ecology, 3(4), 359-366.

Urrego, S. H., \& Gonzáles, A. C. (2000). Estudios ecológicos en el área de influencia del proyecto hidroeléctrico Porce II (pp. 232). Empresas públicas de Medellín. Universidad Nacional de Colombia Sede Medellín.

Vallejo, J. M. I., Londoño-V, A. C., López-C., R., Galeano, G., Álvarez-D. E., \& Devia, A, W. (2005). Establecimiento de parcelas permanentes en bosques de Colombia, Instituto de Investigación en recursos biológicos Alexander von Humboldt (pp. 310). Bogotá D.C., Colombia. (Serie: Métodos para estudios ecológicos a largo plazo; No.1).

Villa, G. A. M. (2005). Caracterización diamétrica de las especies maderables en bosques primarios del cerro Murrucucú (Trabajo de grado). Universidad Nacional de Colombia. Facultad de Ciencias Agropecuarias, sede Medellín.

Zar, J. H. (1999). Biostatistical Analisys (pp. 938). Fourth edition, New Jersey: Prentice Hall Inc. 